\title{
THE TAYLER INSTABILITY AT LOW MAGNETIC PRANDTL NUMBERS: CHIRAL SYMMETRY BREAKING AND SYNCHRONIZABLE HELICITY OSCILLATIONS
}

\author{
F. Stefani*, V. Galindo, A. Giesecke, N. Weber, T. Weier \\ Helmholtz-Zentrum Dresden - Rossendorf, Bautzner Landstr. 400, 01328 Dresden, \\ Germany
}

\begin{abstract}
:
The current-driven, kink-type Tayler instability (TI) is a key ingredient of the Tayler-Spruit dynamo model for the generation of stellar magnetic fields, but is also discussed as a mechanism that might hamper the up-scaling of liquid metal batteries. Under some circumstances, the TI involves a helical flow pattern which goes along with some $\alpha$ effect. Here we focus on the chiral symmetry breaking and the related impact on the $\alpha$ effect that would be needed to close the dynamo loop in the Tayler-Spruit model. For low magnetic Prandtl numbers, we observe intrinsic oscillations of the $\alpha$ effect. These oscillations serve then as the basis for a synchronized Tayler-Spruit dynamo model, which could possibly link the periodic tidal forces of planets with the oscillation periods of stellar dynamos.
\end{abstract}

1. Introduction Current-driven instabilities have been known for a long time in plasma physics [1. A case in point is the so-called $z$-pinch [2, i.e. a straight current $j_{z}$ guided through the plasma which produces an azimuthal magnetic field $B_{\varphi}$. This field is susceptible to both the axisymmetric $(m=0)$ sausage instability and the non-axisymmetric $(m=1)$ kink instability.

In plasmas, the kink instability saturates by two processes which can be interpreted in terms of mean-field MHD. First, the $\beta$ effect leads to a (radially dependent) counter-current which modifies the radial dependence $B_{\varphi}(r)$ so that the (ideal) stability condition $\partial\left(r B_{\varphi}^{2}(r)\right) / \partial r<0[\underline{3}$ becomes marginally fulfilled. Second, the $\alpha$ effect leads to some azimuthal current $j_{\varphi}$, producing a $B_{z}$ component, which contributes to saturation according to the Kruskal-Shafranov condition for the safety parameter. However, the occurrence of a finite value of $\alpha$ is not obvious, since it requires a spontaneous symmetry breaking between a left handed and a right handed TI mode which are, in principle, equally likely [4, 5].

While the magnetic Prandtl number $\mathrm{Pm}=\mu_{0} \sigma \nu$ of fusion plasmas is typically close to one, there are other relevant problems that are characterized by much smaller values. This applies, in particular, to liquid metal batteries [6, 7, , 8, whose upscalability might be limited by the kink-type Tayler instability (TI) 3 . Using a quasi-stationary code 9 on the basis of the OpenFOAM library we have recently shown 10, that the saturation mechanism of the TI changes completely for low Pm: here, the quadratic combination of the $m=1$ velocity perturbations produces $m=0$ and $m=2$ velocity components which suppress the further growth of the TI.

Another interesting effect that was observed in [10] is the occurrence of helicity 
oscillations in the saturated state. While those oscillations are not very interesting for liquid metal batteries, they could be highly relevant for stellar dynamo models of the Tayler-Spruit type [11. The poloidal-to-toroidal field transformation for this type of nonlinear dynamo is easily provided by the usual $\Omega$ effect due to differential rotation, but the toroidal-to-poloidal field transformation requires some $\alpha$ effect to be produced by the TI. If this $\alpha$ effect has a tendency to intrinsic oscillations, this could give a chance for weak external forces (such as exerted by planets) to synchronize the entire dynamo.

This paper summarizes the corresponding ideas as outlined in [10] and [12, and adds a new aspect related to the question of whether this type of synchronized dynamo might provide the correct orientation of the butterfly diagram for sunspots.

2. Helicity waves in the saturated state of the Tayler instability For the sake of concreteness, we study the features of the TI in a cylinder of heightto-diameter ratio $H /(2 R)=1.25$, which is passed through by an axial current. We choose $\mathrm{Pm}=10^{-6}$ and a Hartmann number $\mathrm{Ha}=B_{\varphi} R(\sigma / \rho \nu)^{1 / 2}=100$ which is already significantly higher than the critical Hartmann number $\mathrm{Ha}_{\text {crit }}=21.09$ for the infinitely long cylinder [13].

Figure 1 illustrates the occurrence of helicity oscillations in the saturated regime of the TI. Panel (a) shows the logarithms of the Reynolds number for the total flow and its individual azimuthal modes $m=0,1,2$. The initial exponential increase of the dominant $m=1$ mode is later accompanied by the steeper increase of the nonlinearily produced $m=0$ and $m=2$ modes. Saturation sets in shortly before $t_{n}=0.1$ (the time is normalized to the viscous time scale) when the energy of the $m=0$ and $m=2$ modes becomes comparable to that of the dominant $m=1$ mode. What is interesting is the behavior of the $\alpha$ effect in panel (b), which produces an azimuthal current and, therefore, an axial magnetic field. In the saturated regime (for $t_{n}>0.1$ ), this spontaneous symmetry breaking gives way to a pronounced oscillatory behaviour. In contrast to the oscillation of $\alpha$ and the related helicity, the $\beta$ effect (c) is rather constant in the saturation regime.

An illustration of the helicity oscillation is provided in Fig. 1d which shows the velocity field of the TI at 4 particular instants. While the two main TI vortices, which are typical for the chosen aspect ratio, point essentially in the same direction, we can observe some slight change of their tilt which produces the oscillation of the helicity and $\alpha$.

3. Synchronized helicity oscillations and the solar dynamo cycle The observed helicity oscillation seems to be a generic feature of the saturated TI for large enough Ha at low Pm (note that Bonanno and Guarnieri 14] could not find helicity oscillations in a dissipation-free model). We ask now for its possible implications for Tayler-Spruit type stellar dynamos. Specifically, we will introduce some $m=2$ viscosity perturbation of the base state which serves as a surrogate for the tidal torque of planets on the stellar tachocline. The background of our consideration is the claimed relation of the $\sim 11$ years period of the dominant tidal forces of the Venus-Earth-Jupiter system with the $\sim 22$ years of the solar cycle 15, 16, 17, 18, 19, 20, 21.

Although these tidal forces are usually considered as much too weak to influence the solar dynamo, one should also keep in mind the large gravitational acceleration at the tachocline that amounts to $540 \mathrm{~m} / \mathrm{s}^{2}$ [22]. This translates the apparently tiny tidal heights in the order of $1 \mathrm{~mm}[23$ to equivalent velocities of 

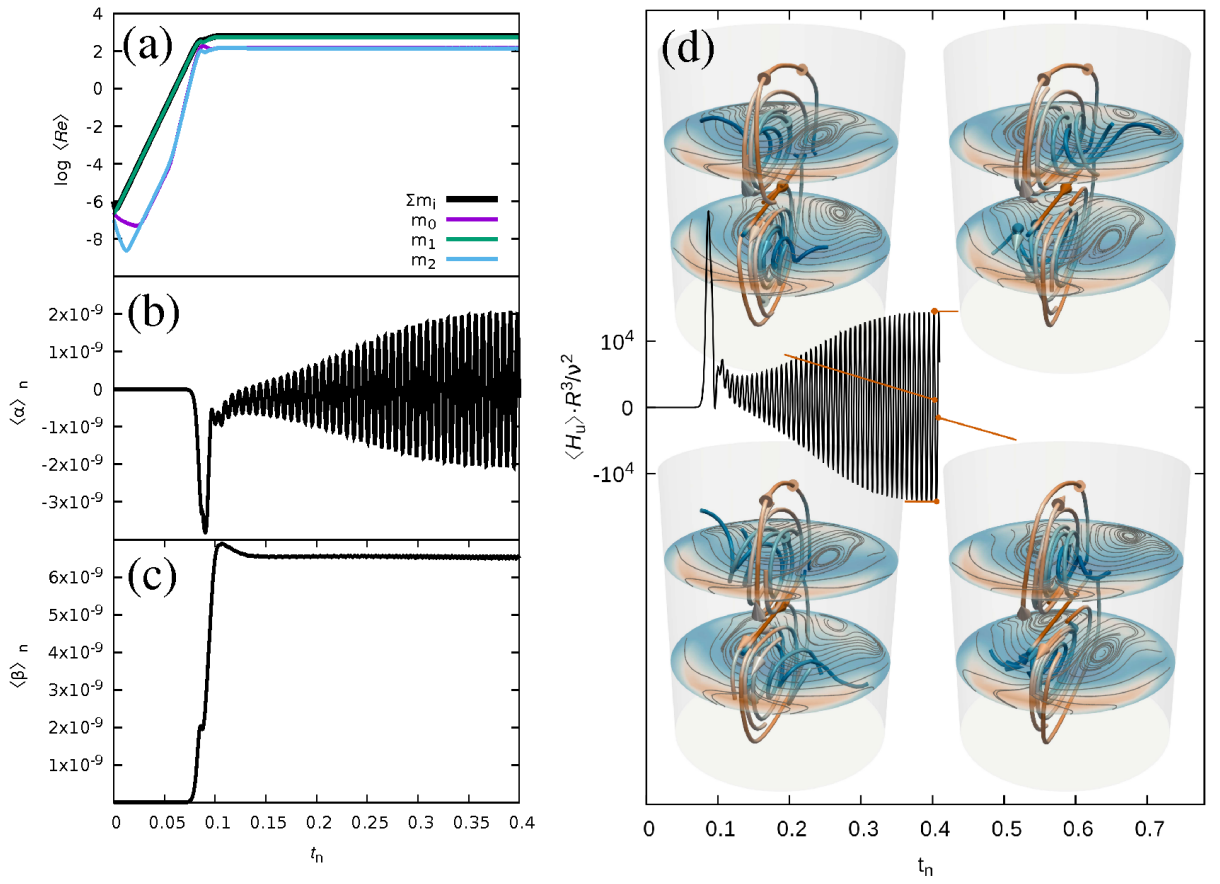

Figure 1: Evolution of various quantities for a developing TI at $\mathrm{Pm}=10^{-6}$ and $\mathrm{Ha}=100$. (a) Reynolds number of the total flow, and of the first azimuthal modes individually. The exponential increase (note the log-scale of the ordinate axis) is followed by a saturated state with a nearly constant Reynolds number. (b) Normalized $\alpha$ effect, showing a spontaneous symmetry breaking in the kinematic growth phase of the TI, and a clear oscillation in the saturated phase. (c) Normalized $\beta$ effect, showing a rather constant value in the saturated regime. (d) Normalized helicity, and four snapshots of the velocity field in the saturation regime. Note the slightly changing tilts of the two vortices which produce the oscillation of the helicity and $\alpha$. After [10]. 

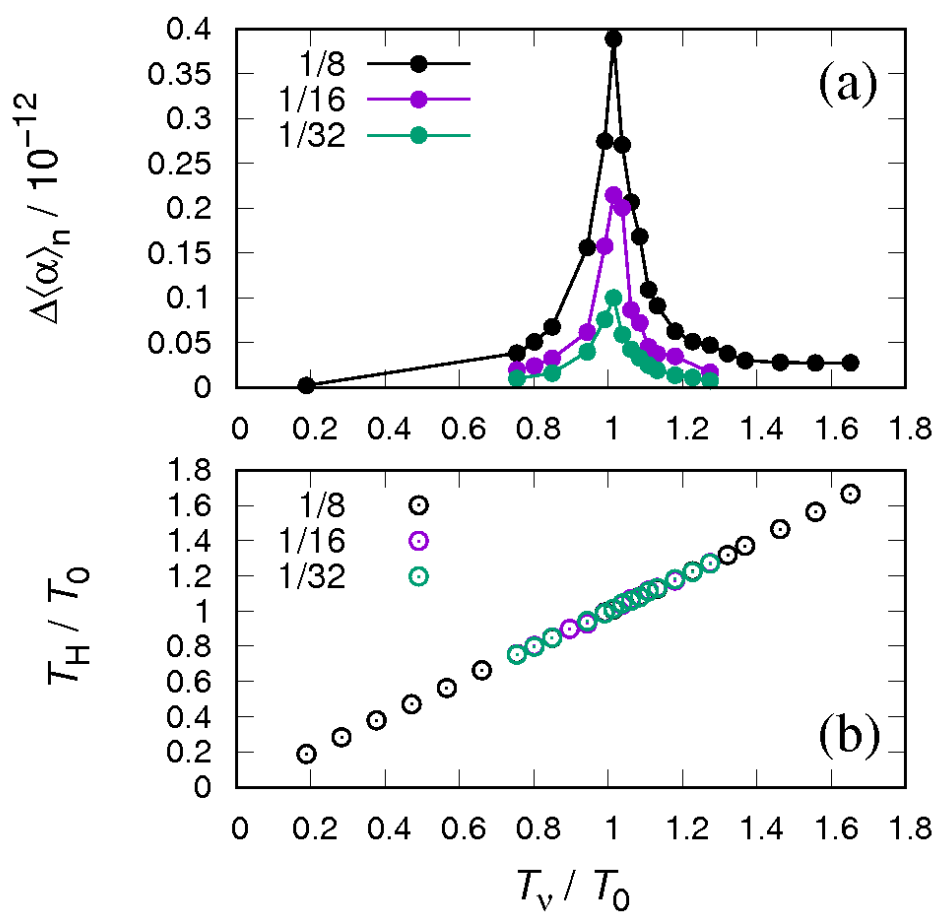

Figure 2: Resonance between an $m=2$ viscosity oscillation according to Eq. (1) and the oscillation of $\alpha$. (a) Amplitude of the $\alpha$ oscillation in dependence on $T_{\nu} / T_{0}$. (b) Ratio $T_{H} / T_{0}$ in dependence on $T_{\nu} / T_{0}$, showing a clear 1:1 resonance. After [12].

$v=(2 g h)^{1 / 2} \sim 1 \mathrm{~m} / \mathrm{s}$. Such velocities, when allowed to coherently develop in the quiet regions of the tachocline (and not being overwhelmed by the highly fluctuating velocities prevailing in the convection zone) might indeed be relevant for the dynamo.

Without any perturbation, when choosing $\mathrm{Ha}=80$, the TI would lead to a weak helicity oscillation with a certain period $T_{0}$. We impose on this state an $m=2$ oscillation of the viscosity $\nu$ in the form

$$
\nu(r, \phi, t)=\nu_{0}\left\{1+A\left[1+0.5 r^{2} / R^{2} \sin (2 \phi)\left(1+\cos \left(2 \pi t / T_{\nu}\right)\right)\right]\right\}
$$

which includes a constant term $\nu_{0}(1+A)$ and an additional term with an $m=2$ azimuthal dependence that is oscillating with a period $T_{\nu}$. For the intensity of the viscosity wave we select now three specific values $A=1 / 32,1 / 16,1 / 8$. The resulting amplitude of the oscillation of $\alpha$ (and the helicity) is shown in Fig. 2a, its period $T_{H}$ in Fig. 2b. Obviously, we obtain a strong 1:1 resonance of the oscillation amplitude at $T_{\nu}=T_{0}$.

What are the possible consequences of this synchronization of $\alpha$ for a complete Tayler-Spruit dynamo? To answer this question we consider the simple zerodimensional equation system

$$
\begin{aligned}
\frac{d a(t)}{d t} & =\alpha(t) b(t)-\tau^{-1} a(t) \\
\frac{d b(t)}{d t} & =\Omega a(t)-\tau^{-1} b(t)
\end{aligned}
$$


which describes the transformation of poloidal field $a$ to toroidal field $b$ via some $\Omega$ effect, and the back-transformation of toroidal to poloidal field via the TI-based $\alpha$ effect. The free decay time of the respective modes is denoted by $\tau$. Note that similarly simple equation systems have been widely used to understand various dynamo features 24. Hereby, $\alpha(t)$ is parametrized according to

$$
\alpha(t)=\frac{c}{1+g b^{2}(t)}+\frac{p b^{2}(t)}{1+h b^{4}(t)} \sin \left(2 \pi t / T_{\nu}\right)
$$

which represents, in its first part, some constant term which is only quenched by magnetic field energy $b^{2}$, plus a time-dependent part with the tidal period $T_{\nu}$, the prefactor of which is chosen such as to emulate the resonance condition $T_{\nu}=T_{0}$ seen in Fig. 2.

The resulting dynamo behaviour is quite interesting. Figure 3a shows approximately half a period of a dynamo cycle for the particular choice $c=0.8, g=1$, $p=8, h=10, \Omega=10$. We observe a clear sign change of the magnetic field, and amazing "spiky" features of $\alpha$ close to the turning point of $a$ and $b$. The capitals A...E mark various instants with specific features to be explained in the following: Initially, at $\mathrm{A}, \alpha$ is strongly quenched by the large value of $b$, while its oscillatory part is negligible since $b$ is so strong that we are far away from resonance. While $b$ decreases, it reaches a level at which the TI helicity oscillation becomes resonant with the viscosity oscillation. This happens at $\mathrm{B}$ when $b \sim 0.56$, which actually corresponds to the maximum of the pre-factor $b^{2} /\left(1+10 b^{4}\right)$ of the oscillatory term in Eq. (4). At this point $\alpha$ becomes strongly negative. Shortly after, at C, $b$ drops to zero, so that the quenching of the constant term of $\alpha$ disappears and $\alpha$ acquires the unquenched value $c$ (here $\sim 0.8)$. Later, at $\mathrm{D}, b$ passes again through the resonant point $b \sim-0.56$ for the helicity oscillation so that the oscillatory part contributes again its large, but now positive, value to $\alpha$. Finally, at E, $b$ increases quite smoothly until it reaches a maximum amplitude where $\alpha$ is strongly quenched and rather constant.

In Fig. 3b we compare an appropriately scaled and time-shifted segment of our $a(t)$ with the available time series of the $20 \mathrm{nHz}$ filtered north and south polar field data. An amazing coincidence exists between the additional peaks of the north and south polar field in Fig. 3(b) and the corresponding spikes of our $a$ (indicated by the three black arrows). Another point, seen in Fig. 3(a), is related to the vigorous, "spiky" variations of $\alpha$ close to the reversal point of $a$ and $b$. It is tempting to relate this behaviour to the short-term sign changes of the current-helicity, as observed recently by [25].

4. And the butterfly diagram? For decades, mean-field theory had served as the standard model of the solar dynamo, providing a natural explanation for the periodicity and the equator-ward sunspot propagation of the solar cycle [26]. However, this model suffered a blow when helioseismology mapped the differential rotation in the solar interior [27]. In particular, the positive radial shear in a $30^{\circ}$ strip around the equator results in a serious problem with the Parker-Yoshimura sign rule that requires $\alpha \partial \Omega / \partial r<0$ in the northern hemisphere for the correct equator-ward propagation of sunspots [28, 29].

A possible solution of this dilemma was found in the Babcock-Leighton mechanism [30, 31, which interprets the generation of poloidal field by the stronger diffusive cancellation of the leading sunspots (closer to the equator) compared with that of the trailing spots (farther from the equator). This leads to a spatially separated, or flux-transport, type of dynamo [32], which also provides the correct butterfly diagram when it is combined with an appropriate meridional circulation. 

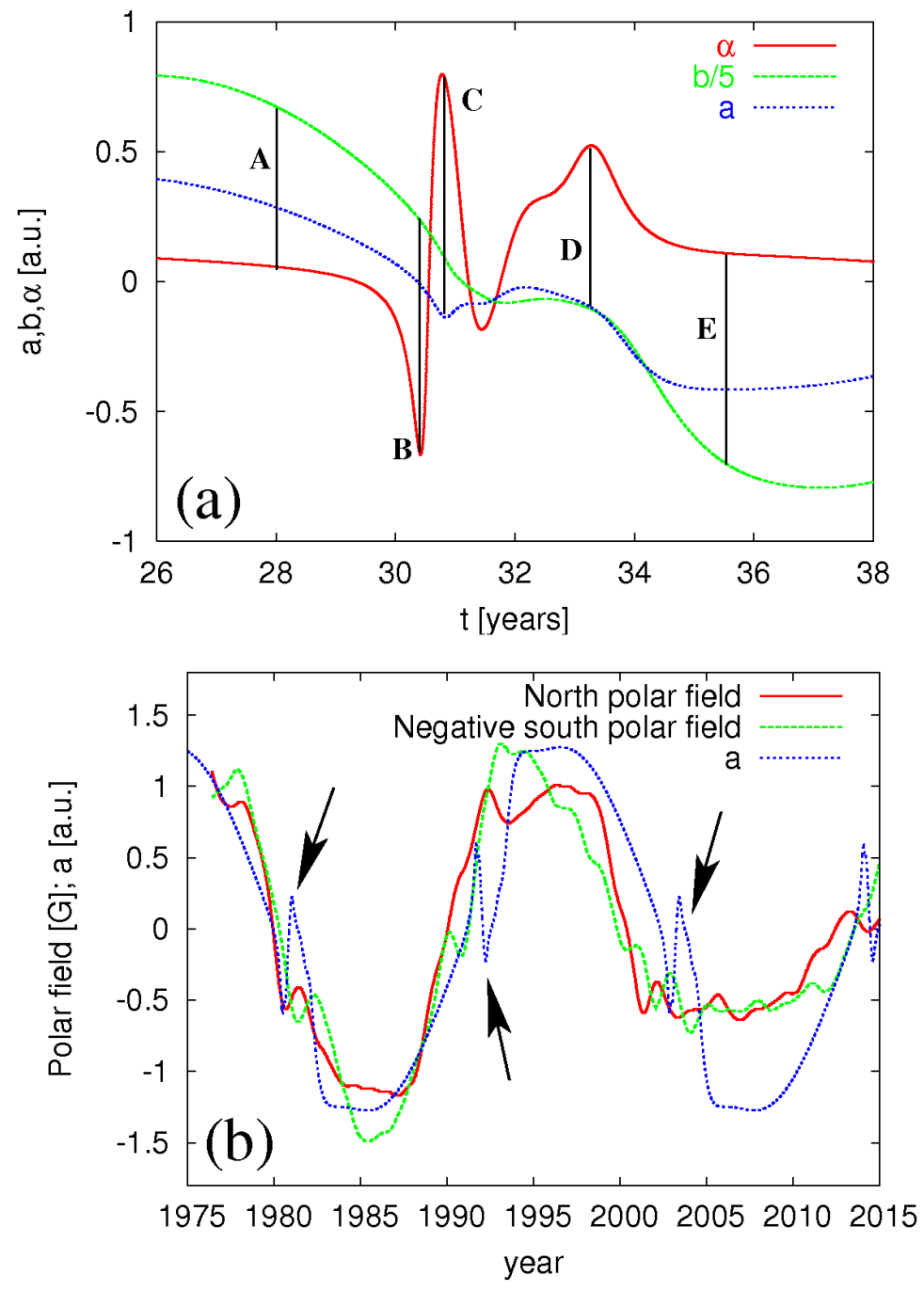

Figure 3: Evolution of the equation system (2-4) with tidal forcing of 11.07 years. (a) Parameter choice $c=0.8, g=1, p=8, h=10, \Omega=10$. (b) Comparison of the north and south polar magnetic field and the parameter $a$, computed for $c=0.8$, $p=8, h=10, \Omega=50, g=1$, appropriately scaled and shifted in time. The field data are the $20 \mathrm{nHz}$ filtered data from Wilcox Solar Observatory (courtesy J.T. Hoeksema). After [12]. 


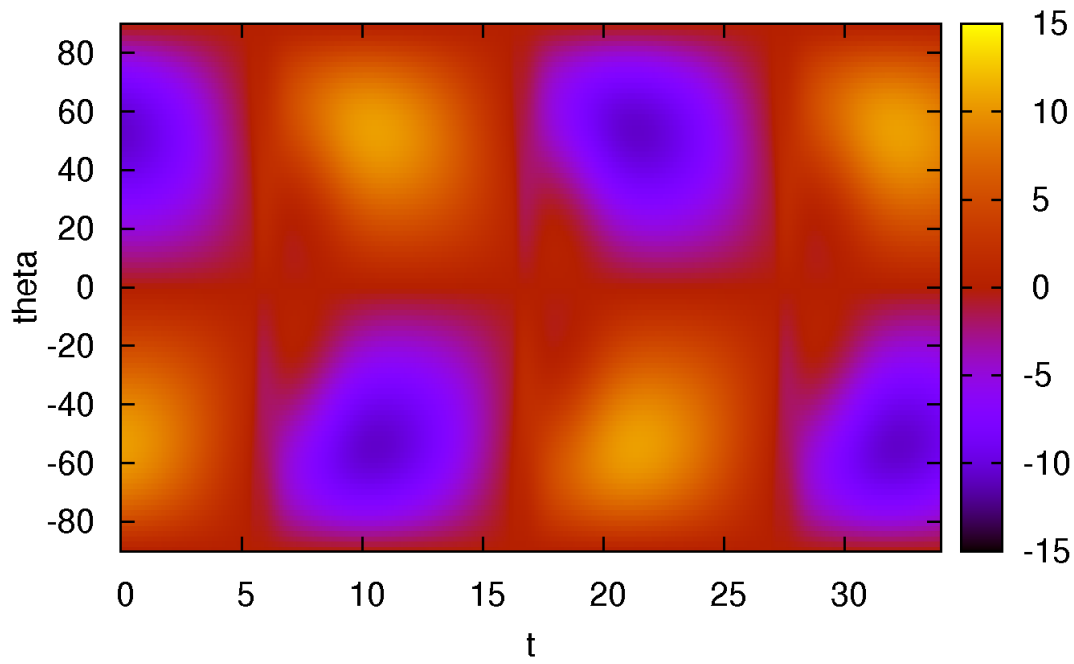

Figure 4: Butterfly diagram resulting from the equation system (5-8) when choosing $\tau_{a_{1}}^{-1}=1, \tau_{a_{2}}^{-1}=3.2, \tau_{b_{1}}^{-1}=1$, and $\tau_{b_{2}}^{-1}=3.2, \kappa=1$, and $c=0.8, g=1, p=8$, $h=10, \Omega=100$, and $T_{0}=11.07 \mathrm{yr}$. Note that, although $c$, i.e. the constant part of $\alpha$, and $\Omega$ are both positive, the butterfly is oriented in the right direction.

Coming back to our synchronization model, we might ask what direction of the butterfly diagram it would provide. For this purpose we consider the following slight extension of the equation system (2-4):

$$
\begin{aligned}
\frac{d a_{1}(t)}{d t} & =\alpha(t) b_{1}(t)-\tau_{a_{1}}^{-1} a_{1}(t) \\
\frac{d a_{2}(t)}{d t} & =\alpha(t)\left(b_{1}(t)+b_{2}(t)\right)-\tau_{a_{2}}^{-1} a(t) \\
\frac{d b_{1}(t)}{d t} & =\Omega\left(a_{1}(t)-\kappa a_{2}(t)\right)-\tau_{b_{1}}^{-1} b_{1}(t) \\
\frac{d b_{2}(t)}{d t} & =\kappa \Omega a_{2}(t)-\tau_{b_{2}}^{-1} b_{2}(t) .
\end{aligned}
$$

Here, the amplitudes $a_{1}$ and $a_{2}$ represent the first and the second possible meridional harmonics of the poloidal field, while $b_{1}$ and $b_{2}$ stand for the first two harmonics of the toroidal field. Although this equation system is motivated by the system given by Nefedov and Sokoloff [33] we keep its pre-factors a bit more generic since we actually do not know the exact meridional dependence of the first two relevant (poloidal and toroidal) eigenmodes.

While in the original paper 33 the free decay rates of the individual modes were derived as $\tau_{a_{1}}^{-1}=1, \tau_{a_{2}}^{-1}=9, \tau_{b_{1}}^{-1}=4$, and $\tau_{b_{2}}^{-1}=16$ (and the factor $\kappa=3$ ), we choose here $\tau_{a_{1}}^{-1}=1, \tau_{a_{2}}^{-1}=3.2, \tau_{b_{1}}^{-1}=1$, and $\tau_{b_{2}}^{-1}=3.2, \kappa=1$.

The resulting butterfly diagram is shown in Fig. 4, when further choosing $c=0.8, g=1, p=8, h=10, \Omega=100$, and $T_{0}=11.07 \mathrm{yr}$. Surprisingly, our synchronization model produces the correct orientation of the butterfly diagram even when the product of $c$ (the constant part of $\alpha$ ) with $\Omega$ is positive! However, given the many parameters that enter the equation system (5-8), together with the complex expression for $\alpha$, more parameter studies are necessary in order to check the robustness of this behaviour. 
5. Conclusions While the traditional explanation of the Hale cycle of the solar magnetic field relies on intrinsic features of the solar dynamo such as the magnetic diffusivity, the amplitudes of $\Omega, \alpha$ and the meridional flow [34, we have focused on a mechanism that could allow for synchronizing the solar dynamo with planetary tides.

Motivated by the spontaneous occurrence of helicity oscillations in the saturated state of the TI as observed in [10, we studied a simplified model for the resonant excitation of those oscillations by a viscosity oscillation with $m=2$ azimuthal dependence that serves as dummy for a tidal forcing. The helicity and $\alpha$ oscillations, thought to be excited by the 11.07 years periodic tide produced by the Venus-Earth-Jupiter system, served as a "clock" for the 22.14 years dynamo cycle of a reduced, zero-dimensional $\alpha-\Omega$ dynamo model. Actually, similar resonance phenomena have been discussed in connection with the swing excitation of galactic dynamos [35] and with the von-Kármán-sodium dynamo experiment [36]. However, it is a key feature of the mechanism discussed here that it requires only weak external perturbations to trigger the helicity oscillations which just reshuffle the energy between left and right handed TI modes. That way the tiny planetary forces might indeed get a chance to synchronize the solar dynamo. By slightly extending the model of [12] we have also shown that our model may lead to the correct orientation of the butterfly diagram.

An obvious next step is related to the question of whether longer periodicities of the solar dynamo, such as the 87-year Gleissberg cycle, the 210-year Suess-deVries cycle, and the 2300-year Hallstatt cycle [37, 38, 39, 40, 41, 42, could also be explained in the framework of the synchronization model. The solution of this problem might have tremendous consequences, in particular, if any of the disputed mechanisms for connecting solar activity and terrestrial climate [43, 44, 45, 46. could be validated.

Acknowledgments This work was supported by the Deutsche Forschungsgemeinschaft in the frame of the SPP 1488 (PlanetMag), as well as by HelmholtzGemeinschaft Deutscher Forschungszentren (HGF) in frame of the Helmholtz alliance LIMTECH. Wilcox Solar Observatory data used in this study was obtained via the web site wso.stanford.edu (courtesy of J.T. Hoeksema). F. Stefani thanks R. Arlt, A. Bonnano, A. Brandenburg, A. Choudhuri, D. Hughes, M. Gellert, G. Rüdiger, and D. Sokoloff for fruitful discussion on the solar-dynamo mechanism. We thank V. Pipin for pointing out a wrong axis labeling in Fig. 7 of [12] which is now corrected in Fig. 3b.

\section{REFERENCES}

1. H. Goedbloed, S. Poedts. Principles of Magnetohydrodynamics (Cambridge University Press, Cambridge, 2004).

2. W.F. Bergerson, D.A. Hannum, C.C. Hegna, R.D. Kendrick, J.S. Sarff, C.B. Forest Onset and saturation of the kink instability in a current-carrying line-tied plasma. Phys. Rev. Lett., vol. 96 (2006), Art. No. 015004.

3. R.J. TAYLER The adiabatic stability of stars containing magnetic fields. Mon. Not. R. Astr. Soc., vol. 161 (1973), p. 365.

4. M. Gellert, G. RÜdiger, R. Hollerbach Helicity and alpha-effect by currentdriven instabilities of helical magnetic fields. Mon. Not. R. Astr. Soc., vol. 414 (2011), p. 2696.

5. A. Bonanno, A. Brandenburg, F. Del Sordo, D. Mitra Breakdown of chiral symmetry during saturation of the Tayler instability. Phys. Rev. E, vol. 86 (2012), Art. No. 016313. 
6. F. Stefani, T. Weier, G. Gundrum, G. Gerbeth: How to circumvent the size limitation of liquid metal batteries due to the Tayler instability. Energy Conv. Manag., vol. 52 (2011), p. 2982.

7. N. Weber, V. Galindo, F. Stefani, T. Weier. Current-driven flow instabilities in large-scale liquid metal batteries, and how to tame them. J. Power Sources, vol. 265 (2014), p. 166.

8. F. Stefani, V. Galindo, C. Kasprzyk, S. Landgraf, M. Seilmayer, M. Starace, N. Weber, T. Weier. Magnetohydrodynamic effects in liquid metal batteries. IOP Conf. Ser.: Mater. Sci. Eng., vol. 143 (2016), Art. No. 012024.

9. N. Weber, V. Galindo, F. Stefani, T. Weier, T. Wondrak Numerical simulation of the Tayler instability in liquid metals. New J. Phys., vol. 17 (2015), Art. No. 043034.

10. N. Weber, V. Galindo, F. Stefani, T Weier. The Tayler instability at low magnetic Prandtl numbers: between chiral symmetry breaking and helicity oscillations. New J. Phys., vol. 15 (2012), Art. No. 113013.

11. H. Spruit Dynamo action by differential rotation in a stably stratified stellar interior. Astron. Astrophys., vol. 381 (1973), p. 923.

12. F. Stefani, A. Giesecke, N. Weber, T. Weier. Synchronized helicity oscillations: a link between planetary tides and the solar cycle? Solar Phys., doi:10.1007/s11207016-0968-0 (2015).

13. W. Herreman, C. Nore, L. Cappanera, J.-L. Guermond Tayler instability in liquid metal columns and liquid metal batteries. J. Fluid Mech., vol. 771 (2015), p. 79 .

14. A. Bonanno, F. Guarnieri On the possibility of helicity oscillations in the saturation of the Tayler instability. arxiv.org/abs/1610.00616 (2016)

15. R. Wolf Extract of a letter to Mr. Carrington. Mon. Not. R. Astron. Soc., vol. 19 (1859), p. 85.

16. C.J. Bollinger A 44.77 year Jupiter-Venus-Earth configuration sun-tide period in solar-climatic cycles. Proc. Okla. Acad. Sci., vol. 33 (1952), p. 307.

17. K. TAKAHASHi On the relation between the solar activity cycle and the solar tidal force induced by the planets. Solar Phys., vol. 3 (1968), p. 598.

18. K. Wood Sunspots and planets. Nature, vol. 240 (1972), p. 91.

19. C.C. Hung Apparent relations between solar activity and solar tides caused by the planets. NASA/TM-2007-214817 (2007).

20. I.R.G. WILsON The Venus-Earth-Jupiter spin-orbit coupling model. Pattern Recogn. Phys., vol. 1 (2013), p. 147.

21. V.P. Okнlopkov The 11-year cycle of solar activity and configurations of the planets. Mosc. U. Phys. Bull., vol. 69 (2014), p. 257.

22. T. Wood The solar tachocline: A self-consistent model of magnetic confinement. (Dissertation, University of Cambridge, 2010).

23. H. Zhang Planetary tides and the sunspot cycles. Solar Phys., vol. 42 (2006), p. 529.

24. A.L. Wilmot-Smith, D. Nandy, G. Hornig, P.C.H. Martens A time delay model for solar and stellar dynamos. Astrophys. J., vol. 652 (2006), p. 696.

25. H. ZhANG ET AL. Current helicity of active regions as a tracer of large-scale solar magnetic helicity. Astrophys. J., vol. 751 (2006), Art. No. 47.

26. M. Steenbeck, F. Krause Zur Dynamotheorie stellarer und planetarer Magnetfelder. I. Berechnung sonnenähnlicher Wechselfeldgeneratoren. Astron. Nachr., vol. 291 (1969), p. 49. 
27. T.M. Brown, J. Christensen-Dalsgand, W.A. Dziembowski, P. Goode, D.O. Gough, C. Morrow Inferring the Sun's internal angular velocity from observed p-mode frequency splitting. Astrophys. J., vol. 343 (1989), p. 526.

28. E.N. PArker Hydromagnetic dynamo models. Astrophys. J., vol. 122 (1955), p. 293.

29. H. Yoshimura Solar-cycle dynamo wave propagation. Astrophys. J., vol. 201 (1975), p. 740 .

30. H.W. BABCOCK The topology of the Sun's magnetic field and the 22-year cycle. Astrophys. J., vol. 133 (1961), p. 572.

31. R.B. Leighton Transport of magnetic field on the Sun. Astrophys. J., vol. 140 (1964), p. 1547.

32. A.R. Choudhuri The solar dynamo with meridional circulation. Astron. Astrophys., vol. 303 (1995), p. L29.

33. S.N. Nefedov, D. Sokoloff Parker's dynamo as specific behaviour of a dynamical system. Astron. Rep., vol. 54 (2010), p. 247.

34. P. Charbonneau, M. Dikpati Stochastic fluctuations in a Babcock-model of the solar cycle. Astrophys. J., vol. 543 (2000), p. 1027.

35. M. ChibA, M.Tosa Swing excitation of galactic magnetic-fields induced by spiral density waves. Mon. Not. Roy. Astron. Soc., vol. 244 (2000), p. 714.

36. A. Giesecke, F. Stefani, J. Burguete Impact of time-dependent nonaxisymmetric velocity perturbations on dynamo action of von Krmn-like flows.. Phys. Rev. E, vol. 86 (2000), Art. No. 066303.

37. J.A. Abreu, J. Beer, A. Ferriz-Mas, K.G. McCracken, F. Steinhilber Is there a planetary influence on solar activity? Astron. Astrophys., vol. 548 (2012), p. A88.

38. I. Charvatova Solar-terrestrial and climatic phenomena in relation to solar inertial motion. Surv. Geophys., vol. 18 (1997), p. 131.

39. P.D. Jose Suns motion and sunspots. Astron. J., vol. 70 (1965), p. 193.

40. M. Palus, J. Kurths, U. Schwarz, D. Novotna, I. Charvatova Is the solar activity cycle synchronized with the solar inertial motion? Int. J. Bifurc. Chaos Appl. Sci. Eng., vol. 10 (2000), p. 2519.

41. N. SCAFETta The complex planetary synchronization structure of the solar system. Pattern Recogn. Phys., vol. 2 (2014), p. 1.

42. N. Scafetta, F Milani, A. Bianchini, S. Ortolani On the astronomical origin of the Hallstatt oscillation found in radiocarbon and climate records throughout the Holocene. Earth Sci. Rev. , vol. 162 (2016), p. 24.

43. H. Svensmark, E. Frits-Christensen Variation of cosmic ray flux and global cloud coverage - A missing link in solar-climate relationships. J. Atmos. Solar-Terr. Phys., vol. 59 (1997), p. 1225.

44. N. ScAfEtTa Empirical evidence for a celestial origin of the climate oscillations and its implications. J. Atmos. Solar-Terr. Phys., vol. 72 (2010), p. 951.

45. L.J. Gray Solar influences on climate. Rev. Geophys., vol. 48 (2010), Art. No. RG4001.

46. F. Gervais Anthropogenic $\mathrm{CO}_{2}$ warming challenged by 60 -year cycle. Earth Sci. Rev., vol. 155 (2016), p. 129. 